\title{
Experimental therapy for colon cancer: Anti-cancer effects of TLR9 agonism, combination with other therapeutic modalities, and dependence upon p53
}

\author{
ELIZABETH R. RAYBURN ${ }^{*}$, WEI WANG ${ }^{*}$, RUIWEN ZHANG ${ }^{1-3}$ and HUI WANG ${ }^{1,2}$ \\ ${ }^{1}$ Department of Pharmacology and Toxicology, Division of Clinical Pharmacology, ${ }^{2}$ Comprehensive Cancer Center \\ and ${ }^{3}$ Gene Therapy Center, University of Alabama at Birmingham, Birmingham, AL, USA
}

Received February 1, 2007; Accepted March 23, 2007

\begin{abstract}
The present study demonstrates the efficacy of utilizing TLR9 (toll-like receptor 9) agonism as a potential therapy for colon cancer. We examined the effects of two types of TLR9 agonists: a traditional CpG oligonucleotide and a novel immunomodulatory oligonucleotide in models of colon cancer, both alone and in combination with conventional cancer therapies. Because the tumor suppressor p53 is involved in many anti-cancer pathways, and is mutated in more than $50 \%$ of cancers, we determined whether p53 is necessary for the antitumor effects observed following treatment with TLR9 agonists. We also established that colon cancer cells express TLR9, which has not been demonstrated previously. The effects of TLR9 agonism on the growth, proliferation and apoptosis of colon cancer cells in vitro was then examined. We report five major discoveries: i) TLR9 agonism results in significant activity in models of colon cancer, ii) TLR9 agonists increase the anti-tumor effects of radiation and chemotherapy, iii) p53 is not required for the anti-cancer effects of TLR9 agonism, iv) human colon cancer cells express TLR9, and v) TLR9 agonism leads to decreased cell survival and proliferation and induces apoptosis of colon cancer cells in vitro. These results provide a basis for future studies determining the potential of utilizing TLR9 agonists for human colon cancer therapy.
\end{abstract}

\section{Introduction}

Colon cancer is the third most common cancer in the USA, and is the second and third leading cause of cancer death in

Correspondence to: Dr Hui Wang, University of Alabama at Birmingham, 1670 University Blvd. VH 112, Birmingham, AL 35294, USA

E-mail: hui.wang@ccc.uab.edu

${ }^{*}$ Contributed equally

Abbreviations: oligo, oligonucleotide; TLR9, toll-like receptor 9

Key words: experimental therapy, oligonucleotide, chemosensitization, radiosensitization men and women, respectively (1). It is estimated that 112,340 new cases of colon cancer will have been diagnosed in the US in 2007, and that more than 52,180 people will have died of the disease (1). Progress has been made in the past few decades due to increased screening and public awareness, as well as because of the many molecular and genetic discoveries made, however, the incidence and mortality of the disease remain high. The predominant treatment strategy for colon cancer is surgical resection, although other therapies, ranging from traditional cytotoxic chemotherapy with oxaliplatin and 5-fluorouracil or irinotecan, to monoclonal antibodies targeting VEGF (vascular endothelial growth factor) or EGFR (epidermal growth factor receptor) are also currently being used. Other specific molecular therapies are in the pipeline, including hormone therapy, therapy targeting growth factors, imatinib mesylate (Gleevec) targeting the BCR-ABL fusion, and therapy with natural compounds such as resveratrol (2). However, despite these advances, colon cancer still has a high mortality rate, and new therapeutic approaches are urgently needed.

Bacterial DNA and synthetic oligonucleotides containing unmethylated $\mathrm{CpG}$ motifs (CpG oligos) activate the immune system via toll-like receptor 9 (TLR9), a pathogen-associated molecular pattern recognition receptor $(3,4)$. TLR9 belongs to a family of proteins that recognize a variety of pathogenic molecules, including lipopolysaccharide (TLR2/4), flagellin (TLR5), dsRNA (TLR3) and of particular interest for this study: bacterial, plasmid and synthetic DNA (TLR9) $(5,6)$. Expression of these receptors has been observed in immune cells, particularly B cells and dendritic cells (3-7), and their stimulation leads to the activation of a complex signaling cascade terminating in $\mathrm{NF}-\kappa \mathrm{B}$ and $\mathrm{AP}-1$ mediated gene expression $(8,9)$. Unlike most of the other TLRs, TLR9 is not expressed at the cell surface. Instead, it is localized within endosomal compartments or the Golgi complex, which may act to prevent its recognition of 'self' DNA (10).

Stimulation of TLR9 with bacterial/plasmid DNA or synthetic $\mathrm{CpG}$ oligos results in the production of various cytokines and chemokines, including IL-12, IL-6, IFN $\alpha / \beta$, and IFN- $\gamma$ (11-13). A number of studies have indicated that administration of TLR9 agonists to animals can lead to antitumor effects including decreased tumor growth, increased animal survival, and in some cases, complete eradication of 
the tumor (14-18). Bacterial DNA, CpG oligos and other TLR9 agonists are currently being investigated in human clinical trials for cancer and other indications (19-21).

TLR9 agonists containing synthetic immunostimulatory motifs and novel DNA structures have been reported $(13,22)$. These novel TLR9 agonists have been the subject of extensive studies of their immunostimulatory profiles, and have demonstrated potent increases in immunostimulatory cytokine and chemokine production (23-28). Moreover, these agents have been shown to have anti-tumor activity in several in vitro and in vivo models of cancer $(18,28)$.

The tumor suppressor p53 is integral to numerous cellular processes, including cell cycle progression and apoptosis, as well as the response to cancer therapies $(29,30)$. More than $50 \%$ of human cancers have mutations to p53 (loss or gain of function), and numerous studies have been accomplished to decrease the activity of mutant $\mathrm{p} 53$, or to increase the activity of wt p53 (29,31). These studies have shown the importance of the molecule and the need for wt p53 expression in order to obtain an optimal therapeutic response $(32,33)$.

In the present study, we evaluated both a $\mathrm{CpG}$-containing oligonucleotide and a novel synthetic TLR9 agonist in models of human colon cancer alone and in combination with conventional cancer therapies. We evaluated the expression of TLR9 within colon cancer cells, and the direct effects of TLR9 agonism on colon cancer cells in vitro. Finally, we investigated the importance of $\mathrm{p} 53$ to the response to therapy with a TLR9 agonist.

\section{Materials and methods}

Reagents and chemicals. All chemicals and solvents were of the highest analytical grade available. Cell culture media, fetal bovine serum (FBS); phosphate-buffered saline (PBS), sodium pyruvate, non-essential amino acids, penicillinstreptomycin and other cell culture supplies were obtained from the Comprehensive Cancer Center Media Preparation Shared Facility, University of Alabama at Birmingham. Taxotere was purchased from Aventis Pharmaceutical Products Inc. (Bridgewater, NJ). Matrigel basement membrane matrix was obtained from Becton-Dickinson Labware (Bedford, MA).

The conventional CpG oligonucleotide - 5'-CTATCTG ACGTTCTCTGT-3' and synthetic TLR9 agonist - 5'-TCTG TCRTTCT-X-TCTTRCTGTCT-5' (where R and X stand for 2'-deoxy-7-deazaguanosine and a glycerol linker, respectively), and a control non-stimulatory oligonucleotide - 5'-TCTCAC CTTCT-X-TCTTCCACTCT-5' were synthesized, purified, and analyzed as previously reported (27).

Cells and culture conditions. The p53 and p21-intact and deficient HCT116 human colon cancer cell lines were kindly provided by Dr Bert Vogelstein (Johns Hopkins Oncology Center, Baltimore, MD). The HCT116 cell lines [parental cell (p53 $\left.3^{+/+}, \mathrm{p} 21^{+/+}\right)$; p21 knockdown (p53 $\left.3^{+/+}, \mathrm{p} 21^{-/-}\right)$; and p53 knockdown (p53-/, p21 $\left.{ }^{+/+}\right)$] were maintained in McCoy's-5A media. The p53 and p21 double knockdown cell line HCT116 $\left(\mathrm{p} 53^{-/-}, \mathrm{p} 21^{-/-}\right.$) was generated in our laboratory using RNAi technology to knockdown p53 in HCT116 (p53+/+, p2 $1^{-/-}$) cells; these cells were cultured in the same medium plus $0.5 \%$ puromycin. The LS174T and DLD-1 colon cancer cell lines were obtained from the American Type Culture Collection (Rockville, MD) and cultured according to their instructions. All media contained $1 \%$ penicillin-streptomycin and $10 \%$ FBS.

Animal tumor models. The animal use and care protocol was approved by the Institutional Animal Use and Care Committee of the University of Alabama at Birmingham. Female athymic pathogen-free nude mice (nu/nu, 4-6 weeks) were purchased from Frederick Cancer Research and Development Center (Frederick, MD) and were fed with commercial diet and provided water ad libitum.

Human cancer xenograft models were established using the methods reported previously (34-36). Briefly, when confluence reached $80 \%$, cultured LS174T, DLD-1 and HCT116 cells were harvested from monolayer cultures, washed with the serum-free medium described above and resuspended in the same medium with Matrigel ${ }^{\circledR}$ basement membrane matrix at a 3:1 ratio, then injected subcutaneously (s.c.) into the left inguinal area of nude mice. All animals were monitored for activity, physical condition, body weight, and tumor growth. Tumor growth was determined by caliper measurement in two perpendicular diameters of the implant every other day. Tumor weight (in g) was calculated by the formula, $1 / 2 a \times b^{2}$ where ' $a$ ' is the long diameter and ' $b$ ' is the short diameter (in $\mathrm{cm}$ ).

Treatment of animals with oligonucleotide alone or in combination with chemotherapy. The animals bearing human cancer xenografts were randomly divided into various treatment groups and a control group (5-10 mice/group). The untreated control group received physiological saline $(0.9 \% \mathrm{NaCl})$ only. The oligonucleotides dissolved in physiological saline $(0.9 \% \mathrm{NaCl})$ were administered by s.c. injection at doses of $0.1-2 \mathrm{mg} / \mathrm{kg} / \mathrm{day}, 3$ doses per week. The treatment schedule was as follows: $L S 174 T$ and $D L D-1$ models: the $\mathrm{CpG}$ oligo and synthetic TLR9 agonist were administered by s.c. injection at a dose of $0.1,1.0$ or $2.0 \mathrm{mg} / \mathrm{kg}$ three doses per week beginning when palpable tumors were established (mean tumor size $91 \mathrm{mg}$ ). For the combination treatment study in the LS174T model, the CpG oligo or synthetic TLR9 agonist was administered by s.c. injection at a dose of $1.0 \mathrm{mg} / \mathrm{kg}$ on days $0,2,4,7,9$ and 11. Paclitaxel was given by intraperitoneal (i.p.) injection at $10 \mathrm{mg} / \mathrm{kg}$ on days 0 and 3. Taxotere was given by i.p. injection at $15 \mathrm{mg} / \mathrm{kg}$ on days 0 and 7 . HCT116 model: each of the four HCT116 cell lines had a different genetic background, and the parental $\left(\mathrm{p} 53^{+/+}, \mathrm{p} 21^{+/+}\right)$, p53 knockdown, p21 knockdown and p53/p21 double knockdown cells were used to generate in vivo xenograft tumors. When palpable tumors were established (mean tumor size $77 \mathrm{mg}$ ), the synthetic TLR9 agonist and control oligo were given at $1 \mathrm{mg} / \mathrm{kg}$ by s.c. injection, 3 doses per week for 3 weeks.

In vivo combination treatment with the synthetic TLR9 agonist and radiation. Mice bearing HCT116 xenografts (parental and p53 knockdown cells) were randomly divided into multiple treatment groups, in addition to a control group (5 mice/group). The synthetic TLR9 agonist was selected for these studies based upon its greater activity. The TLR9 agonist ( $1 \mathrm{mg} / \mathrm{kg}$ ) and control oligo were administered following the same 
treatment protocol as above. Mice receiving radiation treatments were first anesthetized with 70-100 $\mu$ l of a mixture of ketamine $(20 \mathrm{mg} / \mathrm{ml})$ and xylazine $(20 \mathrm{mg} / \mathrm{ml})$ at a $6.7: 1$ ratio and then placed under a lead shield designed to expose only the tumors to the radiation beam. $\gamma$-irradiation was administered by a ${ }^{60} \mathrm{Co}$ Picker unit irradiator (JL Shepard Co.; Glendale, CA) $(1.56 \mathrm{~Gy} / \mathrm{min})$. Animals received $10 \mathrm{~Gy}$ of radiation on days 3 and 10. Mice in the combination treatment groups were pre-treated with the TLR9 agonist or the control oligo $4 \mathrm{~h}$ prior to $\gamma$-irradiation.

Detection of TLR9 in cancer cells. HCT116 parental and derivative cells and DLD-1 cells were evaluated for TLR9 mRNA and protein expression according to previously described protocols (37). In brief, total RNA was extracted with the TRIzol reagent (Invitrogen) and used as a template to generate cDNA. The TLR9 cDNA was specifically amplified using primers as reported by Bauer et al (38). The TLR9 and ß-actin protein levels were evaluated by Western blotting using an anti-TLR9 antibody from Calbiochem/EMD Biosciences (Cat no. OP185) following procedures described previously $(35,36)$.

Cell survival assay. The percentage of cell survival (HCT116 parental and p53 kDa) was determined using the MTT assay. The cells were exposed to the synthetic TLR9 agonist or the control oligo $(0,1,5,10,50,100 \mathrm{nM})$ with or without Lipofectin (Life Technologies, Gaithersburg, MD; $7 \mu \mathrm{g} / \mathrm{ml}$ ) for $48 \mathrm{~h}$. The cell survival percentages were calculated by dividing the mean OD of TLR9 agonist-containing wells by that of control wells. Three separate experiments were accomplished.

Detection of apoptosis. Cells in early and late stages of apoptosis were detected with an Annexin V-FITC apoptosis detection kit from BioVision (Mountain View, CA). Cells were transfected with the TLR9 agonist $(100 \mathrm{nM})$ or control oligo (100 $\mathrm{nM})$ and incubated for $24 \mathrm{~h}$ prior to analysis. Media and cells were collected and washed with serum-free media. Cells were then re-suspended in Annexin $\mathrm{V}$ binding buffer followed by addition of Annexin V-FITC and propidium iodide (PI). The samples were incubated in the dark for $5 \mathrm{~min}$ at room temperature prior to analysis. Samples were analyzed using a Becton-Dickinson FACSCalibur instrument (Ex = $488 \mathrm{~nm} ; \mathrm{Em}=530 \mathrm{~nm}$ ). Cells that were positive for Annexin V-FITC alone (early apoptosis) and Annexin V-FITC and PI (late apoptosis) were counted.

Cell proliferation assay. BrdUrd incorporation into cells was accomplished using a cell proliferation assay kit from Oncogene (La Jolla, CA). Cells were seeded in 96-well plates and transfected with the TLR9 agonist (100 $\mathrm{nM})$ or the control oligo $(100 \mathrm{nM})$ for $24 \mathrm{~h}$. BrdUrd was added to the medium $10 \mathrm{~h}$ before terminating the treatment. The level of BrdUrd incorporated into cells was quantified by anti-BrdUrd antibody, and absorbance was measured at dual wavelengths of $450 / 540 \mathrm{~nm}$ with an OPTImax microplate reader (Molecular Devices, Sunnyvale, CA).

Data and statistical analysis. The anti-tumor activity (as measured by differences in tumor mass) was expressed as mean

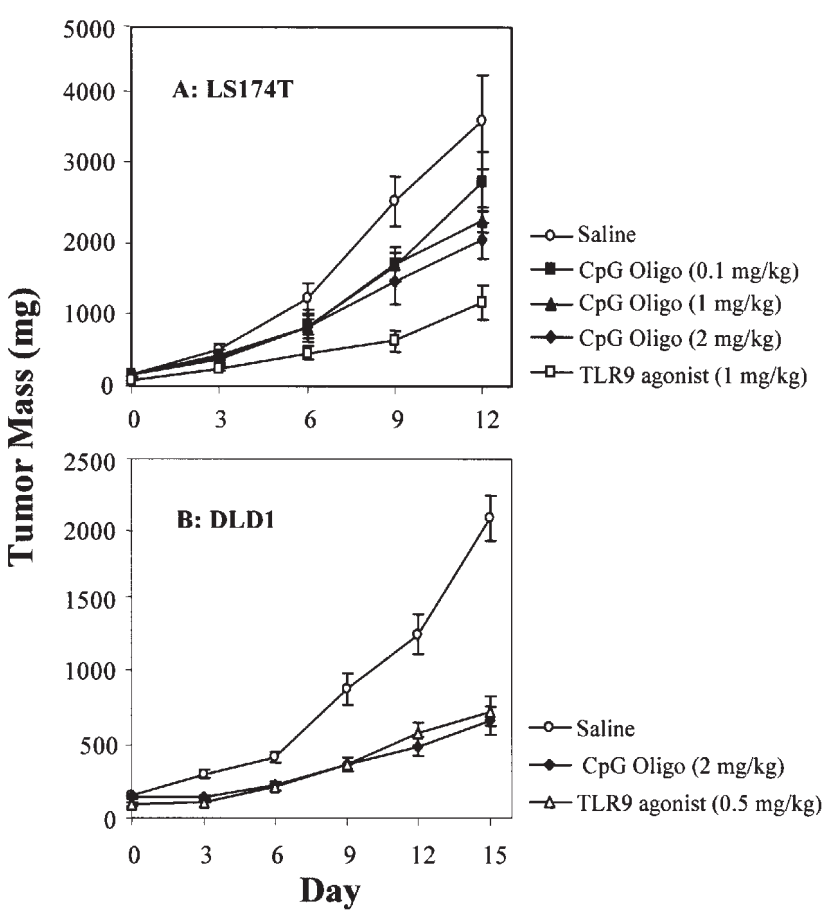

Figure 1. TLR9 agonism decreases the growth of xenograft colon cancer tumors. A CpG oligonucleotide or a synthetic TLR9 agonist was administered to nude mice bearing LS174T or DLD-1 human colon cancer xenografts. (A) Effects of the CpG oligo and TLR9 agonist in the LS174T model. (B) Effects of the CpG oligo and TLR9 agonist in the DLD-1 model. The compounds were dissolved in sterile physiological saline $(0.9 \% \mathrm{NaCl})$ and administered by s.c. injection at a dose of $0.1-2.0 \mathrm{mg} / \mathrm{kg} / \mathrm{day}, 3 \mathrm{doses}$ per week.

and standard deviations, and the significance of differences was analyzed by $\chi^{2}$ and ANOVA analyses.

\section{Results}

TLR9 agonism decreases the growth of xenograft colon tumors. Both the CpG oligo and the synthetic TLR9 agonist had a significant inhibitory effect $(\mathrm{P}<0.05)$ on tumor growth in nude mice bearing human colon cancer LS174T or DLD-1 xenografts. In the LS174T model, the CpG oligo inhibited tumor growth at all doses administered, and demonstrated a trend toward a dose-response. Mice receiving $0.1 \mathrm{mg} / \mathrm{kg} /$ day 3 times/week showed tumor growth inhibition by $24 \%$ on day 12 , those receiving $1.0 \mathrm{mg} / \mathrm{kg} /$ day showed $37 \%$ tumor growth inhibition, and those receiving $2.0 \mathrm{mg} / \mathrm{kg} /$ day showed $45 \%$ tumor growth inhibition (Fig. 1A). The synthetic TLR9 agonist demonstrated greater anti-tumor effects. Administration of $1.0 \mathrm{mg} / \mathrm{kg}$ /day of the TLR9 agonist three times/week led to $>68 \%$ inhibition of tumor growth on day 12 after initiating treatment (Fig. 1A).

To demonstrate that the effects of the $\mathrm{CpG}$ oligo and synthetic TLR9 agonist were not confined to the LS174T model, another model of human colon cancer with a different genetic background was used. Animals bearing DLD-1 xenograft tumors showed a similar response to treatment with the CpG oligo and TLR9 agonist as did animals bearing LS174T tumors. The CpG oligo inhibited tumor growth by $67 \%$, while the TLR9 agonist inhibited tumor growth by $65 \%$ 


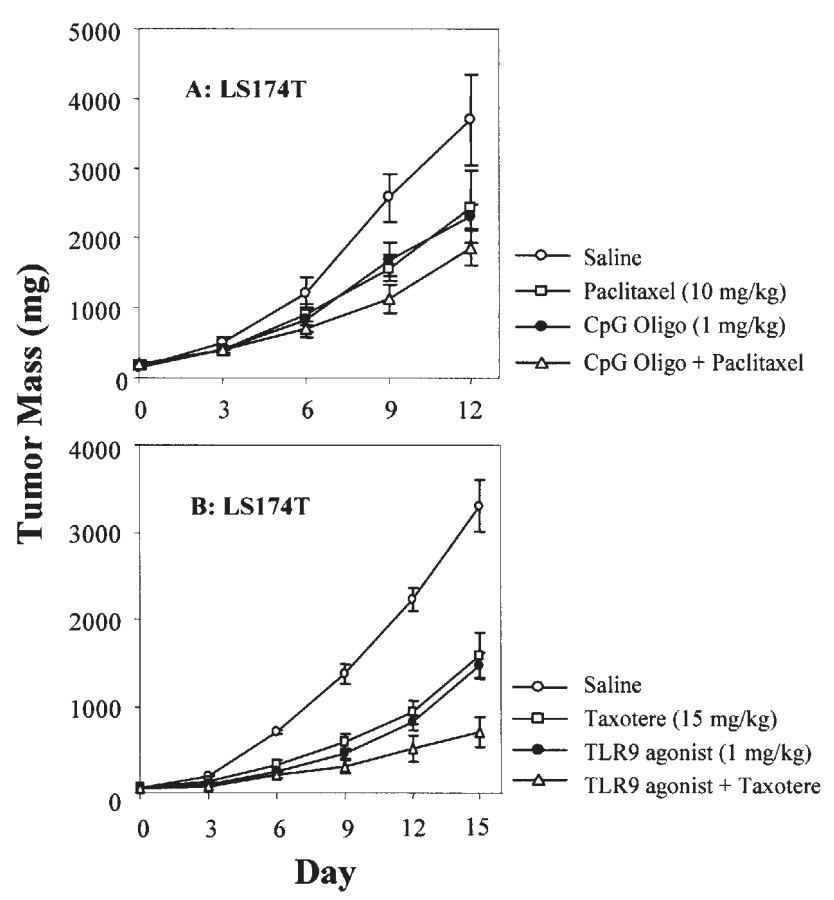

Figure 2. The CpG oligonucleotide (A) and TLR9 agonist (B) further decrease the growth of tumors treated with the chemotherapeutic agents paclitaxel and taxotere in the LS174T model.

(Fig. 1B) on day 15. Notably, a lower dose of the synthetic TLR9 agonist was required to achieve a similar effect (65 and $67 \%$ growth inhibition) as the CpG oligo.
TLR9 agonism acts in concert with conventional chemotherapy. Current cancer therapy in the clinic typically centers on the use of two or more agents in combination. In order to investigate whether a TLR9 agonist could be combined with a chemotherapeutic agent to further decrease tumor growth, we combined the $\mathrm{CpG}$ oligo and synthetic TLR9 agonist with the chemotherapeutic agents paclitaxel and taxotere. Combining taxane chemotherapy with TLR9 agonism led to significant inhibition of the growth of LS174T colon cancer xenograft tumors $(\mathrm{P}<0.01)$. The $\mathrm{CpG}$ oligo led to an increase in the anti-tumor efficts of paclitaxel on day 12, increasing tumor growth inhibition from 34 (paclitaxel alone) to 50\% (combination of the two agents) (Fig. 2A). Growth of tumors from the group receiving both the TLR9 agonist and taxotere was inhibited by $\sim 80 \%$, while tumor growth from groups treated with the TLR9 agonist or taxotere alone was inhibited by 56 and $52 \%$, respectively (Fig. 2B). These results confirm that a TLR9 agonist can be combined with chemotherapy to improve the anti-cancer response.

The TLR9 agonist inhibits the growth of colon cancer xenograft tumors, independent of p53. p53 is an important cell cycle regulatory molecule that mediates the response of normal and tumor tissue to stress and treatment with various agents $(33,39,40)$. Moreover, p53 is frequently mutated in human cancers, suggesting that many tumors do not respond normally to treatment with cytotoxic and therapeutic agents. Having observed that the TLR9 agonist was effective alone and in combination with taxotere, and having observed no significant differences in the response to the TLR9 agonist in the two

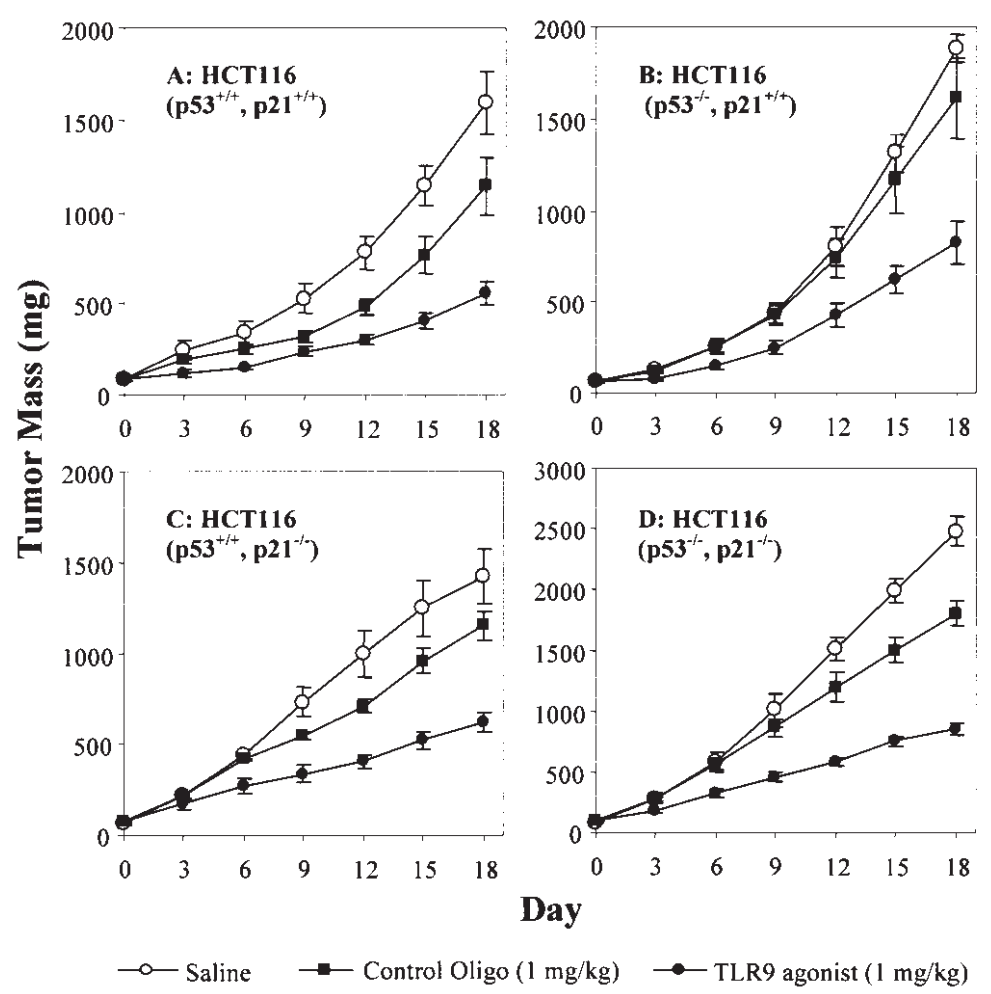

Figure 3. The anti-tumor effects of TLR9 agonism do not depend on the p53 status of the tumors. The TLR9 agonist (1 mg/kg) and control oligo ( $1 \mathrm{mg} / \mathrm{kg}$ ) were administered to nude mice bearing HCT-116 human colon cancer xenografts with different genetic backgrounds of p53 and p21. (A), HCT116 parental

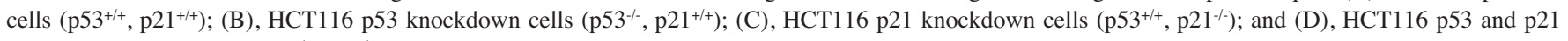
double knockdown cells $\left(\mathrm{p} 53^{-/-}, \mathrm{p} 21^{-/-}\right)$. 


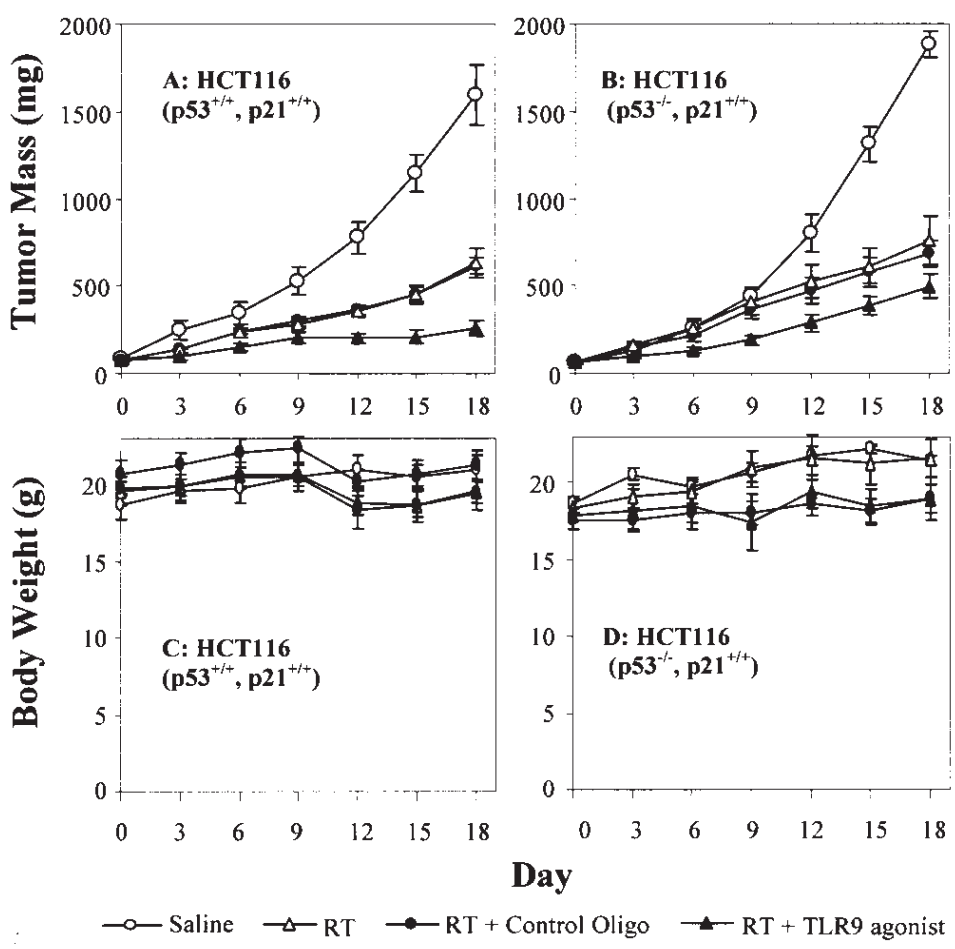

Figure 4. The TLR9 agonist sensitizes colon xenograft tumors to radiation treatment. Both the HCT116 p53 wild-type (p53 $3^{+/+}$, p21 $1^{+/+}$) (A) and HCT116 p53 knockdown ( $\left.553^{-/-}, \mathrm{p} 21^{+/+}\right)$(B) tumors showed substantial inhibition of growth when treated with a combination of the TLR9 agonist (1 mg/kg) and radiation (10 Gy). There was no significant decrease in body weight for either model (C and D).

xenograft tumor types, we further evaluated the effects of the TLR9 agonist in another model of colon cancer to confirm its anticancer effects and determine whether p53 is important for the effects of the TLR9 agonist. The synthetic TLR9 agonist was evaluated in HCT116 xenograft models with differential expression of both p53 and p21 in order to confirm that wildtype p53 is not required. Cells with different expression of p53 and p21 [parental HCT116 cells (p53+/+, p21 ${ }^{+/+}$); p53

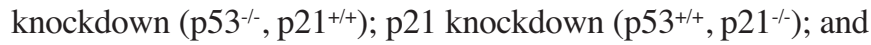
p53 and p21 double knockdown (p53 $\left.3^{--}, \mathrm{p} 21^{--}\right)$] were derived from the parental cell line and used to generate xenograft models. Similar to the results obtained in the studies of LS174T and DLD-1 colon cancer xenograft models, the TLR9 agonist showed a significant inhibitory effect on tumor growth $(\mathrm{P}<0.05)$, with tumor growth inhibition between 56 and $66 \%$ on day 18 days after initiating treatment, depending on the cell line. The control oligo had minimal to modest effects on tumor growth. Neither the expression of p53 nor its downstream target, p21, appears to have an impact on colon cancer tumor growth inhibition induced by TLR9 agonist (Fig. 3). The parental and double knock-down cells showed almost identical growth inhibition, and the single knockdown ( $\mathrm{p} 53^{-/-}$or $\mathrm{p} 21^{-/}$) cells showed a similar but slightly smaller decrease in tumor growth.

The synthetic TLR9 agonist leads to increased anti-tumor activity when combined with radiation. Two of the HCT116 cell lines with different backgrounds of p53 were used to determine the effects of combining TLR9 agonism with radiotherapy. Although the experiments in the different HCT116 cells indicated that p53 is not involved in the anti-cancer effects of TLR9 agonism, we utilized two of these lines because p53 is known to be involved in the cellular response to DNA damage, including that caused by radiation $(33,39,40)$. In the HCT116 parental (p53+/+, p21 $1^{+/+}$) tumor model, the TLR9 agonist or radiation treatment alone caused $65 \%$ and $60 \%$ tumor growth inhibition on day 18 , respectively. However, combining the TLR9 agonist with radiation significantly increased the tumor growth inhibition $(\mathrm{P}<0.01$, Fig. $4 \mathrm{~A})$, inhibiting tumor growth by $84 \%$, a $24 \%$ increase over radiation alone. In the HCT116 p53 knockdown (p53/-, p $21^{+/+}$) model, combining TLR9 agonism with radiation caused a change from 60 to $77 \%$ tumor growth inhibition $(\mathrm{P}<0.01$, Fig. 4B). In both models, the control oligo had no additional inhibitory effect on tumor growth in radiationtreated animals. Radiation-treated animals had a slight (but not significant) loss in body weight (Fig. 4C and D for the p53 wt and kd cells, respectively). Combining the synthetic TLR9 agonist with radiation therapy did not lead to additional loss of body weight. These results suggest that combining a TLR9 agonist with radiation could provide a novel way to increase the anti-tumor response. It may also allow for the administration of lower doses of radiation, which would greatly improve patient quality of life.

TLR9 is expressed in human colon cancer cells. Since we observed potent anti-tumor effects in immunocompromised nude mice, it is possible that a complete immune response is not necessary for TLR9-mediated anti-tumor effects. While the immune system has long been thought to be the major mediator of the effects of TLR9 agonists, it is possible that there may be other mechanisms of action for these oligo- 


\section{A: TLR9 mRNA}

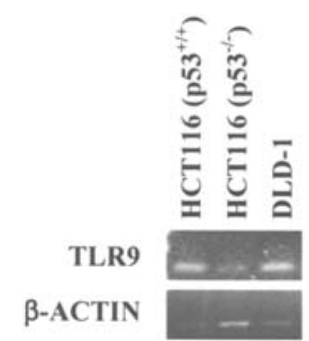

C: $\underline{\mathrm{HCT} 116\left({\mathrm{p} 53^{+/+}}^{+/} \mathrm{p} 21^{+/+}\right)}$
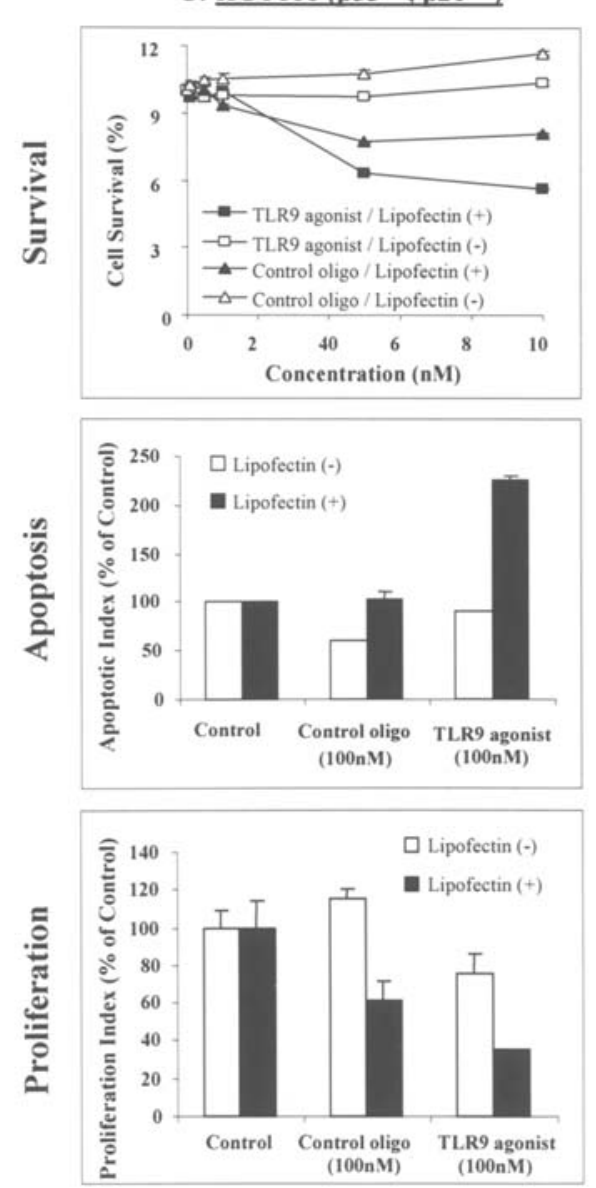

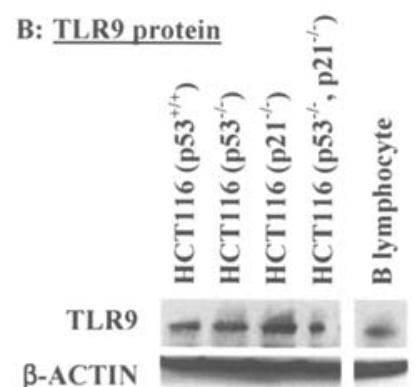

D: $\mathrm{HCT} 116\left(\mathrm{p5} 3^{-/}, \mathrm{p} 21^{+/+}\right)$
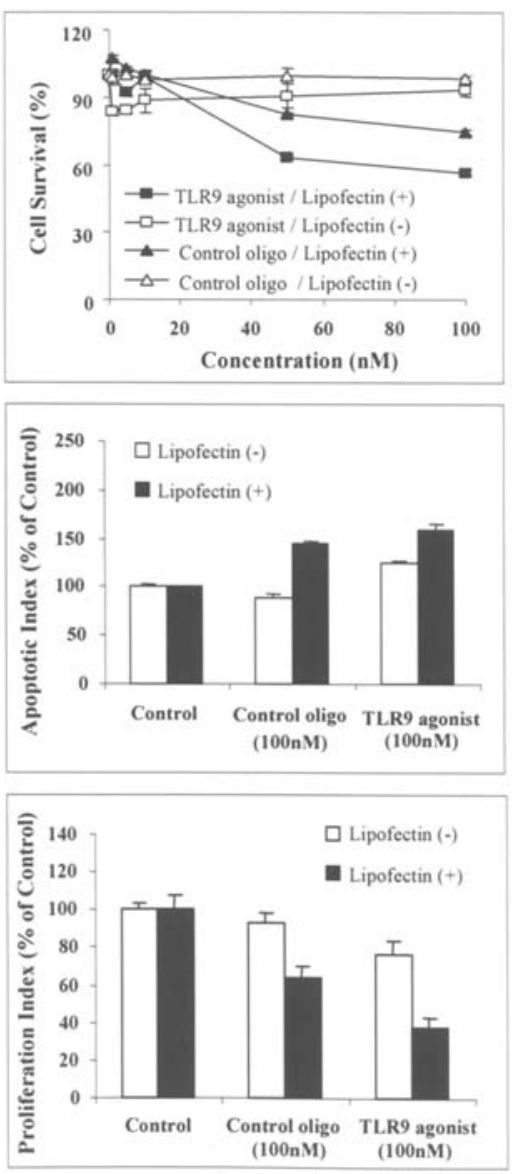

Figure 5. TLR9 mRNA (A) and protein (B) are expressed in human colon cancer cells. TLR9 agonism affects colon cancer cell survival, apoptosis and proliferation $\left[\mathrm{C}, \mathrm{HCT} 116\left(\mathrm{p} 53^{+/+}, \mathrm{p} 21^{+/+}\right)\right.$; D, HCT116 $\left.\left(\mathrm{p} 53^{-/+}, \mathrm{p} 21^{+/+}\right)\right]$. In the presence or absence of Lipofectin, cells were incubated with the TLR9 agonist (100 nM) or control oligo $(100 \mathrm{nM})$ for $24 \mathrm{~h}$ for measurement of apoptosis and proliferation and for $48 \mathrm{~h}$ for evaluation of cell survival. Top panels (cell survival), cells in 96-well plates were exposed to the TLR9 agonist or to the control oligo; the percentage of surviving cells was determined using the MTT assay. Middle panel (apoptosis), cells that stained positive for Annexin V-FITC (early apoptosis) and positive for FITC and propidium iodide (late apoptosis) were counted. Relative levels of apoptotic indices were expressed as a percentage of the Lipofectin control. Bottom panel (proliferation), the effects of the TLR9 agonist and control oligo on the proliferation of cells were determined by BrdUrd incorporation using the same treatment protocol as above.

nucleotides. We hypothesized that cancer cells themselves are able to respond to TLR9 agonism. We examined several of the cell lines (HCT116 p53 wt and knockout and DLD-1) that were used in the present studies for expression of TLR9. In these cell lines, we observed that both TLR9 mRNA (Fig. 5A) and protein (Fig. 5B) were expressed.

The synthetic TLR9 agonist decreases survival, increases apoptosis and decreases proliferation in colon cancer cells in vitro. Following our observation of activity in nude mice and based on the fact that colon cancer cells express TLR9, we examined the effects of TLR9 agonism on colon cancer cells in vitro. Two of the colon cancer cell lines used in the present in vivo studies were exposed to increasing concentrations of the TLR9 agonist or control oligo in vitro, with or without the transfection reagent, Lipofectin. These colon cancer cell lines [Fig. 5C, HCT 116 (p53 wt), Fig. 5D, HCT 116 (p53 knockdown)] demonstrated increased apoptosis, decreased cell proliferation and decreased cell survival following treatment with the TLR9 agonist in the presence of Lipofectin. The control oligo had a modest effect. The fact that Lipofectin increased the in vitro effects suggests that the lipid may 
enhance the delivery of the TLR9 agonist to the endosomal compartment, where TLR9 is localized (10).

\section{Discussion}

While some of the newer colon cancer therapies have shown promising results in preliminary studies, and molecular advances such as the discovery of the Wnt/APC pathway provide a means to individually tailor screening protocols $(41,42)$, colon cancer still has a high mortality rate, and new therapeutic approaches are needed. In particular, therapies with minimized side-effects are needed.

TLR9 agonists (including both bacterial preparations and synthetic oligonucleotides) have been used for more than 20 years as therapies for cancer and a variety of other diseases (14-18,43-51). The effects of TLR9 agonism on the immune system have been well-characterized $(5,9,11-13,23-26)$, and TLR9 agonism has been shown to decrease tumor growth and bring about the rejection of a variety of tumors (14-18, $37,43-45,47)$. In the present study, we have demonstrated several important findings. Both the $\mathrm{CpG}$ oligo and the novel synthetic TLR9 agonist showed potent anti-tumor effects against various colon cancer xenograft models, although a lower dose of the novel TLR9 agonist was required to achieve the anti-tumor effects. TLR9 agonism improved the efficacy of treatment with conventional chemotherapeutic agents and radiation therapy, without any change in the toxicity profiles of the CpG oligo or TLR9 agonist, chemotherapy or radiotherapy. This demonstrates that TLR9 agonism can be used in combination with conventional cancer therapies to increase tumor destruction. Perhaps the most interesting of our findings, we have demonstrated that TLR9 is expressed in human colon cancer cells, and that in vitro treatment of cells with a TLR9 agonist results in decreased proliferation and cell survival, and increased apoptosis in a dose-dependent manner. It must be kept in mind that these are cultured human cancer cells, which may or may not be representative of human tumors. Future studies of human tumor and normal tissue specimens need to be accomplished to confirm that TLR9 is expressed in colon tumors.

Additionally, although p53 is a molecule integral in numerous processes regulating cell differentiation, apoptosis and the response to DNA damage, and is mutated or inactivated in more than $50 \%$ of cancers $(29-31,52)$, it does not appear to play a major role in the apoptosis induced by the synthetic TLR9 agonist. Although p53 knockdown HCT116 cells displayed slightly decreased apoptosis in vitro compared to the p53 wild-type parental cells, the difference was not significant. Since growth was inhibited in p53 wild-type (LS174T and HCT116 parental), mutant (DLD-1) and knockdown (HCT116 $\mathrm{p} 53^{-/}$) xenograft tumors, expression of p53 does not appear to affect the response to the synthetic agonist. Evaluation of cells with differential expression of $\mathrm{p} 21^{\mathrm{WAF} 1 / \mathrm{CIP} 1}$, a gene downstream of p53 that regulates cyclin-dependent kinase function (53), demonstrated that the TLR9 agonist was effective for inhibiting the growth of both p21 wild-type and knockdown tumors. Thus, neither p53 nor p21 appears to be important for the effects of TLR9 agonism on tumor cells growth.

Although it could be suggested that the nude mouse xenograft models used for our studies could lead us to under- estimate the effects of TLR9 agonism, other investigators have previously shown that TLR9 agonists can affect xenograft tumor growth $(28,37,54)$. In our studies, we observed potent anti-tumor effects in all three nude mouse xenograft models of colon cancer that were used. While it is true that nude mice are immunocompromised, they do still possess natural killer cells, dendritic cells, and macrophages, and have been shown to have increased serum cytokine levels following treatment with immunostimulatory and immunomodulatory oligonucleotides (55-57). Therefore, it may be that TLR9 agonists can act via the remaining immune cells in the nude mice. On the other hand, it is possible that tumor cells may be directly affected by these agents. We and other investigators have observed that non-immune tissues, including lung cancer cells, express TLR9 $(37,54,58,59)$. In support of this hypothesis, we demonstrated expression of TLR9 in two cultured human colon cancer cell lines, and that the HCT116 wild-type and p53 knockdown cells are affected by the synthetic TLR9 agonist in vitro, in the presence of Lipofectin, in the absence of any immune cells. These results will need to be examined in-depth in future studies to determine if and how cancer cells and tumors are directly affected by TLR 9 agonists.

There have been several pre-clinical studies of $\mathrm{CpG}$ oligos in models of colon cancer. However, most of these models have focused primarily on the use of these oligos to stimulate dendritic cells $(17,60-62)$ or for use as adjuvants for cancer vaccines $(63,64)$. Of the remaining studies, peritumoral and intratumoral injections of $\mathrm{CpG}$ oligos were most frequently examined (65-67). While effective, this type of therapy is unlikely to be applicable in a clinical setting. Two more recent studies examined combining TLR9 agonists with other conventional cancer therapies $(28,37,68)$. Our results support the observations reported in these publications; showing that there is increased anti-tumor activity, low toxicity and improved survival in animals receiving treatment with a TLR9 agonist in combination with conventional cancer therapy.

In conclusion, we have demonstrated that TLR9 agonism results in potent anti-tumor activity against models of colon cancer, independent of their p53 status. Moreover, combining a TLR9 agonist with conventional anticancer therapies may increase tumor cell death above what can be achieved through the use of either therapy alone. These studies provide a rationale for developing TLR9 agonists for use as therapy against colon cancer, either as monotherapy or in combination with conventional therapies.

\section{Acknowledgements}

This study was supported by a research agreement between the University of Alabama (UAB) at Birmingham and Idera Pharmaceuticals, Inc., and by funds from the UAB Comprehensive Cancer Center Cancer Pharmacology Laboratory. The apoptosis analyses were performed by the Flow Cytometry Core of the Arthritis and Musculoskeletal Center, which is supported in part by an NIH grant (P60 AR20614). E.R. Rayburn is supported in part by the USA Department of Defense Prostate Cancer Research Program (grant number W81XWH-06-1-0063). We thank Drs Robert B. Diasio and Donald L. Hill for helpful discussions. 


\section{References}

1. American Cancer Society: Cancer Facts \& Figures 2007 Atlanta, GA: American Cancer Society, 2007. Also available online: http://www.cancer.org/docroot/STT/content/STT_ 1x_Cancer_Facts_Figures_2007/.asp

2. U.S. National Institutes of Health. http://www .clinicaltrials.gov/ (2006) A service of the U.S. National Institutes of Health Developed by the National Library of Medicine.

3. Janeway CA Jr and Medzhitov R: Innate immune recognition. Annu Rev Immunol 20: 197-216, 2002.

4. Hemmi H, Takeuchi O, Kawai T, et al: A Toll-like receptor recognizes bacterial DNA. Nature 408: 740-745, 2000.

5. Pasare $\mathrm{C}$ and Medzhitov R: Toll-like receptors: linking innate and adaptive immunity. Adv Exp Med Biol 560: 11-18, 2005

6. Sandor F and Buc M: Toll-like receptors. I. Structure, function and their ligands. Folia Biol 51: 148-157, 2005.

7. Fillatreau S and Manz RA: Tolls for B cells. Eur J Immunol 36: 798-801, 2006.

8. Kawai T and Akira S: TLR signaling. Cell Death Differ 13: 816-825, 2006.

9. Takeshita S, Gursel I, Ishii KJ, Suzuki K, Gursel M and Klinman DM: Signal transduction pathways mediated by the interaction of CpG DNA with Toll-like receptor 9. Semin Immunol 16: 17-22, 2004.

10. Barton GM, Kagan JC and Medzhitov R: Intracellular localization of Toll-like receptor 9 prevents recognition of self DNA but facilitates access to viral DNA. Nat Immunol 7: 49-56, 2006.

11. Pisetsky DS: Immune activation by bacterial DNA: a new genetic code. Immunity 5: 303-310, 1999.

12. Klinman DM, Yi AK, Beaucage SL, Conover J and Kreig AM: $\mathrm{CpG}$ motifs present in bacteria DNA rapidly induce lymphocytes to secrete interleukin 6 , interleukin 12 , and interferon gamma. Proc Natl Acad Sci USA 93: 2879-2883, 1996.

13. Agrawal S and Kandimalla ER: Modulation of Toll-like receptor 9 responses through synthetic immunostimulatory motifs of DNA. Ann NY Acad Sci 1002: 30-42, 2003.

14. Moore M, Lawrence N and Nisbet NW: Tumour inhibition mediated by BCG in immunosuppressed rats. Int $\mathrm{J}$ Cancer 15: 897-911, 1975.

15. Lin J, Lin E and Nemunaitis J: Bacteria in the treatment of cancer. Curr Opin Mol Ther 6: 629-639, 2004.

16. Carpentier AF, Auf G and Delattre JY: CpG-oligonucleotides for cancer immunotherapy: review of the literature and potential applications in malignant glioma. Front Biosci 8: e115-127, 2003.

17. Heckelsmiller K, Beck S, Rall K, et al: Combined dendritic celland $\mathrm{CpG}$ oligonucleotide-based immune therapy cures large murine tumors that resist chemotherapy. Eur J Immunol 32: 3235-3245, 2002.

18. Wang D, Li Y, Yu D, Song SS, Kandimalla ER and Agrawal S: Immunopharmacological and antitumor effects of secondgeneration immunomodulatory oligonucleotides containing synthetic CpR motifs. Int J Oncol 24: 901-908, 2004.

19. No Authors Listed (PMID 16922592): CpG 7909: PF 3512676 , PF-3512676. Drugs R D. 7: 312-316, 2006.

20. Switaj T and Lasek W: Technology evaluation: HYB-2055, Hybridon. Curr Opin Mol Ther 7: 376-383, 2005.

21. www.clinicaltrials.gov

22. Agrawal S and Kandimalla ER: Role of Toll-like receptors in antisense and siRNA (corrected). Nat Biotechnol 22: 1533-1537, 2004.

23. Yu D, Kandimalla D, Bhagat L, Tang JY, Cong Y, Tang J and Agrawal S: Immunomers'-novel 3'-3'-linked CpG oligodeoxyribonucleotides as potent immunomodulatory agents. Nucleic Acids Res 30: 4460-4469, 2002.

24. Kandimalla ER, Bhagat L, Yu D, Cong Y, Tang J and Agrawal S: Conjugation of ligands at the 5'-end of CpG DNA affects immunostimulatory activity. Bioconjug Chem 13: 966-974, 2002.

25. Kandimalla ER, Bhagat L, Wang D, et al: Divergent synthetic nucleotide motif recognition pattern: design and development of potent immunomodulatory oligodeoxyribonucleotide agents with distinct cytokine induction profiles. Nucleic Acids Res 31: 2393-2400, 2003.

26. Kandimalla ER, Bhagat L, Zhu FG, et al: A dinucleotide motif in oligonucleotides shows potent immunomodulatory activity and overrides species-specific recognition observed with $\mathrm{CpG}$ motif. Proc Natl Acad Sci USA 100: 14303-14308, 2003.
27. Kandimalla ER, Bhagat L, Li Y, et al: Immunomodulatory oligonucleotides containing a cytosine-phosphate-2'-deoxy-7deazaguanosine motif as potent toll-like receptor 9 agonists. Proc Natl Acad Sci USA 102: 6925-6930, 2005.

28. Damiano V, Caputo R, Bianco R, et al: Novel toll-like receptor 9 agonist induces epidermal growth factor receptor (EGFR) inhibition and synergistic antitumor activity with EGFR inhibitors. Clin Cancer Res 12: 577-583, 2006.

29. Lane DP: p53, guardian of the genome. Nature 358: 15-16, 1992.

30. Poyurovsky MV and Prives C: Unleashing the power of p53: lessons from mice and men. Genes Dev 20: 125-131, 2006.

31. Bartek J, Bartkova J, Vojtesek B, Staskova Z, Rejthar A, Kovarik J and Lane DP: Patterns of expression of the p53 tumour suppressor in human breast tissues and tumours in situ and in vitro. Int J Cancer 46: 839-844, 1990.

32. Wiman KG: Strategies for therapeutic targeting of the p53 pathway in cancer. Cell Death Differ 13: 921-926, 2006.

33. Munro AJ, Lain S and Lane DP: P53 abnormalities and outcomes in colorectal cancer: a systematic review. Br J Cancer 92: 434-444, 2005.

34. Wang H, Cai Q, Zeng X, Yu D, Agrawal S and Zhang R: Antitumor activity and pharmacokinetics of a mixed-backbone antisense oligonucleotide targeted to the RI alpha subunit of protein kinase A after oral administration. Proc Natl Acad Sci USA 96: 13989-13994, 1999 .

35. Zhang Z, Li M, Wang H, Agrawal S and Zhang R: Antisense therapy targeting MDM2 oncogene in prostate cancer: effects on proliferation, apoptosis, multiple gene expression, and chemotherapy. Proc Natl Acad Sci USA 100: 11636-11641, 2003.

36. Wang H, Yu D, Agrawal S and Zhang R: Experimental therapy of human prostate cancer by inhibiting MDM2 expression with novel mixed-backbone antisense oligonucleotides: in vitro and in vivo activities and mechanisms. Prostate 54: 194-205, 2003.

37. Rayburn ER, Wang W, Zhang Z, Li M, Zhang R and Wang H: Experimental therapy of prostate cancer with an immunomodulatory oligonucleotide: effects on tumor growth, apoptosis, proliferation, and potentiation of chemotherapy. Prostate 66: 1653-1663, 2006.

38. Bauer S, Kirschning CJ, Hacker H, et al: Human TLR9 confers responsiveness to bacterial DNA via species-specific CpG motif recognition. Proc Natl Acad Sci USA 98: 9237-9242, 2001.

39. Oren M: Relationship of p53 to the control of apoptotic cell death. Semin Cancer Biol 5: 221-227, 1994.

40. Bristow RG, Benchimol S and Hill RP: The p53 gene as a modifier of intrinsic radiosensitivity: implications for radiotherapy. Radiother Oncol 40: 197-223, 1996.

41. Goyle S and Maraveyas A: Chemotherapy for colorectal cancer. Dig Surg 22: 401-414, 2005.

42. McWilliams RR and Erlichman C: Novel therapeutics in colorectal cancer. Dis Colon Rectum 48: 1632-1650, 2005.

43. Tokunaga T, Yamamoto H, Shimada S, et al: Antitumor activity of deoxyribonucleic acid fraction from Mycobacterium bovis BCG. I. Isolation, physicochemical characterization, and antitumor activity. J Natl Cancer Inst 72: 955-962, 1984.

44. Kataoka T, Yamamoto S, Yamamoto T, Kuramoto E, Kimura Y, Yano $\mathrm{O}$ and Tokunaga T: Antitumor activity of synthetic oligonucleotides with sequences from cDNA encoding proteins of Mycobacterium bovis BCG. Jpn J Cancer Res 83: 244-247, 1992.

45. Krieg AM, Yi AK and Hartmann G: Mechanisms and therapeutic applications of immune stimulatory CpG DNA. Pharmacol Ther 84: 113-120, 1999.

46. Zimmermann S, Egeter O, Hausmann S, Lipford GB, Rocken M, Wagner $\mathrm{H}$ and Heeg $\mathrm{K}$ : $\mathrm{CpG}$ oligodeoxynucleotides trigger protective and curative Th1 responses in lethal murine leishmaniasis. J Immunol 160: 3627-3630, 1998.

47. Wang H, Rayburn E and Zhang R: Synthetic oligodeoxynucleotides containing deoxycytidyl-deoxyguanosine dinucleotides (CpG ODNs) and modified analogs as novel anticancer therapeutics. Curr Pharm Des 11: 2889-2907, 2005.

48. Dittmer U and Olbrich AR: Treatment of infectious diseases with immunostimulatory oligodeoxynucleotides containing $\mathrm{CpG}$ motifs. Curr Opin Microbiol 6: 472-477, 2003.

49. Racila DM and Kline JN: Perspectives in asthma: molecular use of microbial products in asthma prevention and treatment. J Allergy Clin Immunol 116: 1202-1205, 2005.

50. Wooldridge JE and Weiner GJ: CpG DNA and cancer immunotherapy: orchestrating the antitumor immune response. Curr Opin Oncol 15: 440-445, 2003. 
51. Ulevitch RJ: Therapeutics targeting the innate immune system. Nat Rev Immunol 4: 512-520, 2004.

52. Lain S and Lane D: Improving cancer therapy by non-genotoxic activation of p53. Eur J Cancer 39: 1053-1060, 2003.

53. Gartel AL, Serfas MS and Tyner AL: p21-negative regulator of the cell cycle. Proc Soc Exp Biol Med 213: 138-149, 1996.

54. Pratesi G, Petrangolini G, Tortoreto M, et al: Therapeutic synergism of gemcitabine and CpG-oligodeoxynucleotides in an orthotopic human pancreatic carcinoma xenograft. Cancer Res 65: 6388-6393, 2005.

55. Li Y, Kandimalla ER, Yu D and Agrawal S: Oligodeoxynucleotides containing synthetic immunostimulatory motifs augment potent Th1 immune responses to HBsAg in mice. Int Immunopharmacol 5: 981-991, 2005.

56. Sparwasser T, Vabulas RM, Villmow B, Lipford GB and Wagner H: Bacterial CpG-DNA activates dendritic cells in vivo: $\mathrm{T}$ helper cell-independent cytotoxic $\mathrm{T}$ cell responses to soluble proteins. Eur J Immunol 30: 3591-3597, 2000.

57. Buhtoiarov IN, Lum HD, Berke G, Sondel PM and Rakhmilevich AL: Synergistic activation of macrophages via CD40 and TLR9 results in T cell independent antitumor effects. J Immunol 176: 309-318, 2006.

58. Pedersen G, Andresen L, Matthiessen MW, Rask-Madsen J and Brynskov J: Expression of Toll-like receptor 9 and response to bacterial CpG oligodeoxynucleotides in human intestinal epithelium. Clin Exp Immunol 141: 298-306, 2005.

59. Droemann D, Albrecht D, Gerdes J, et al: Human lung cancer cells express functionally active Toll-like receptor 9. Respir Res 6: $1,2005$.

60. Brunner C, Seiderer J, Schlamp A, et al: Enhanced dendritic cell maturation by TNF-alpha or cytidine-phosphate-guanosine DNA drives T cell activation in vitro and therapeutic anti-tumor immune responses in vivo. J Immunol 165: 6278-6286, 2000.
61. Okano F, Merad M, Furumoto K and Engleman EG: In vivo manipulation of dendritic cells overcomes tolerance to unmodified tumor-associated self antigens and induces potent antitumor immunity. J Immunol 174: 2645-2652, 2005.

62. Bourquin C, Schreiber S, Beck S, Hartmann G and Endres S: Immunotherapy with dendritic cells and $\mathrm{CpG}$ oligonucleotides can be combined with chemotherapy without loss of efficacy in a mouse model of colon cancer. Int J Cancer 118: 2790-2795, 2006.

63. Saha A, Baral RN, Chatterjee SK, et al: CpG oligonucleotides enhance the tumor antigen-specific immune response of an antiidiotype antibody-based vaccine strategy in CEA transgenic mice. Cancer Immunol Immunother 55: 515-527, 2006.

64. Daftarian P, Song GY, Ali S, Faynsod M, Longmate J, Diamond DJ and Ellenhorn JD: Two distinct pathways of immuno-modulation improve potency of p53 immunization in rejecting established tumors. Cancer Res 64: 5407-5414, 2004.

65. Heckelsmiller K, Rall K, Beck S, et al: Peritumoral CpG DNA elicits a coordinated response of CD8 T cells and innate effectors to cure established tumors in a murine colon carcinoma model. J Immunol 169: 3892-3899, 2002.

66. Sharma S, Karakousis CP, Takita H, Shin K and Brooks SP: Intra-tumoral injection of $\mathrm{CpG}$ results in the inhibition of tumor growth in murine Colon-26 and B-16 tumors. Biotechnol Lett 25: 149-153, 2003.

67. Sharma S, Karakousis CP, Takita H, Shin K and Brooks SP: Cytokines and chemokines are expressed at different levels in small and large murine colon-26 tumors following intratumoral injections of CpG ODN. Neoplasia 6: 523-528, 2004.

68. Balsari A, Tortoreto M, Besusso D, et al: Combination of a CpG-oligodeoxynucleotide and a topoisomerase I inhibitor in the therapy of human tumour xenografts. Eur J Cancer 40: 1275-1281, 2004. 